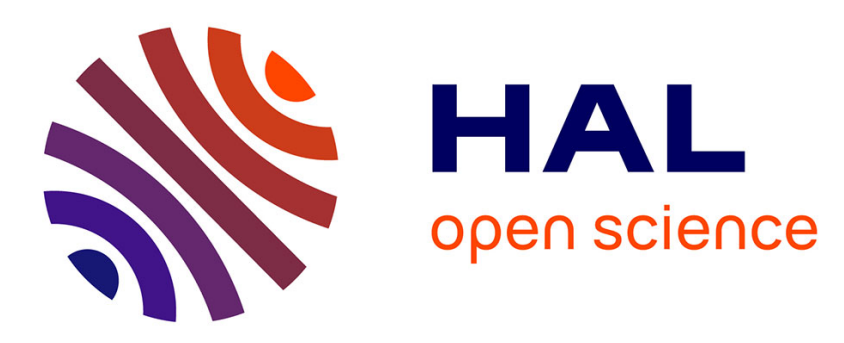

\title{
On Maximum D2D Multiplexing in Asynchronous Communications
}

\author{
Mylene Pischella, Rostom Zakaria, Didier Le Ruyet
}

\section{To cite this version:}

Mylene Pischella, Rostom Zakaria, Didier Le Ruyet. On Maximum D2D Multiplexing in Asynchronous Communications. 2018 IEEE 87th Vehicular Technology Conference (VTC Spring), Jun 2018, Porto, Portugal. pp.1-5, 10.1109/VTCSpring.2018.8417573 . hal-02446290

\section{HAL Id: hal-02446290 https://hal-cnam.archives-ouvertes.fr/hal-02446290}

Submitted on 19 Mar 2020

HAL is a multi-disciplinary open access archive for the deposit and dissemination of scientific research documents, whether they are published or not. The documents may come from teaching and research institutions in France or abroad, or from public or private research centers.
L'archive ouverte pluridisciplinaire HAL, est destinée au dépôt et à la diffusion de documents scientifiques de niveau recherche, publiés ou non, émanant des établissements d'enseignement et de recherche français ou étrangers, des laboratoires publics ou privés. 


\title{
On maximum D2D multiplexing in asynchronous communications
}

\author{
Mylene Pischella, Rostom Zakaria and Didier Le Ruyet \\ CNAM CEDRIC/LAETITIA \\ 292 rue Saint-Martin - 75141 Paris Cedex 03, France \\ Email: mylene.pischella@cnam.fr; rostom.zakaria@cnam.fr; didier.le_ruyet@cnam.fr;
}

\begin{abstract}
This paper studies two different multiplexing problems for Device to Device (D2D) asynchronous communications with different multi-carrier modulations. Several D2D pairs may be multiplexed on the same Resource Blocks (RB) if they are jointly able to reach a high Signal to Interference plus Noise Ratio (SINR). In asynchronous communications, inter-channel interference (ICI) is present on top of co-channel interference, and the multiplexing problem can consequently not be split per $\mathrm{RB}$ since $\mathrm{RB}$ are no longer orthogonal. In this paper, we derive the system model that takes into account ICI and then study the maximum multiplexing problem. Two heuristics based on the Frobenius and the infinity norms that lead to lower bounds on the multiplexing gain are proposed. Then, the maximum sum SINR problem is studied with the infinity norm, showing that higher rates are achieved with Filter Bank Multi-Carrier (FBMC) modulation than with Orthogonal Frequency Division Multiplex (OFDM).
\end{abstract}

\section{INTRODUCTION}

Device to Device (D2D) transmissions are new means to achieve very high individual and sum data rates through multiplexing on the same frequency resources. Devices of the same pair benefit from low propagation losses but some interfering D2D pairs may be close to each others. Consequently, it is necessary to find resource allocation algorithms that determine which D2D pairs should be multiplexed on which Resource Blocks (RB). These algorithms should lead to good trade-offs between frequency orthogonality and full frequency reuse.

In this paper, we focus on the resource allocation problem for D2D pairs when interferences are received asynchronously at each receiver. It is assumed that there is no global synchronization reference, and that devices may start their transmission independently. Then device receivers not only have to cope with co-channel interference from the devices that are multiplexed in the same $\mathrm{RB}$, but also from inter-channel interference (ICI) coming from adjacent RB. Because of ICI, resource allocation cannot be performed independently per RB, but must take into consideration the whole bandwidth. This generates additional complexity and requires new techniques, since this issue has not yet been studied in the literature.

When ICI is not taken into account, the D2D multiplexing problem has been investigated under the mathematical framework of graph coloring in several recent papers [1]-[3]. The vertices of the graph are D2D pairs, and an edge joins two vertices if two D2D transmitters are not allowed to transmit in the same RB because they would highly interfere each other's receivers. In [1], the edges in the graph are built depending on the distance between D2D pairs and from D2D pairs to cellular users. A weighted graph is defined in [2]. The weight of an edge represents the interference between two vertices $k$ and $j$, summed over both transmit-receive $k$ to $j$ and $j$ to $k$ directions. A heuristic is then proposed to maximize the sum rate of cellular and D2D pairs, that iteratively selects the best clustering in the graph and optimizes D2D power allocation for a given clustering. An iterative algorithm based on branch-andbound has also been proposed in [3] when only one D2D pair can be multiplexed with a cellular user per RB. Graph-coloring was also used in our previous letter [4] for RB allocation in asynchronous D2D communications, but this letter focused on power allocation and did not propose new techniques for D2D multiplexing.

On the contrary, this paper is fully dedicated to the D2D multiplexing problem subject to a minimum target Signal to Interference plus Noise Ratio (SINR) per subcarrier. Since the initial problem is too complex, we determine two lower bounds on the initial problem, one of them leading to a distributed solution. The proposed algorithms are valid whether transmission is synchronous or not, and their performance is evaluated with two multi-carrier modulations with different ICI characteristics: Orthogonal Frequency Division Multiplex (OFDM) and Filter Bank Multi-Carrier (FBMC) modulation. The paper is organized as follows: section II introduces the system model and provides a summary of the per-RB allocation system model derived in [4]. Then Section III determines two D2D multiplexing heuristics for maximum multiplexing and Section IV extends the second methodology to the sum SINR maximum problem. The performance results of these algorithms are compared in section $\mathrm{V}$ and conclusions are given in Section VI.

\section{SYSTEM MODEL}

The studied scenario is D2D overlay, with $K$ autonomous D2D pairs sharing a bandwidth $B$ which is different from the cellular bandwidth. The bandwidth is separated in $N$ RB composed of $M$ adjacent subcarriers, with $L=M \times N$ the number of subcarriers. We assume that all D2D transmitters are synchronous with their receiver, and asynchronous with any other receiver. This is due to the varying propagation durations from any transmitter to any other receiver, that may be larger than the cyclic prefix in OFDM. Then, asynchronous interferences are not fully handled by the cyclic prefix, and ICI appears.

Based on prior work from [5], ICI is modelled as interference weights to apply on the power vector. In each subcarrier, all D2D receivers are subject to co-channel as well as interchannel interference. We assume that bandwidth and power allocations is performed per RB. However, ICI is defined per subcarrier. In our previous letter [4], we have derived a system 
model's formulation that allows to take into account the perRB allocation constraint and ICI, and still obtain a simple expression of the data rates. In this paper however, we use the per-subcarrier model at first, and then include the per-RB allocation constraint directly in the RB allocation algorithm.

The ICI weights are modelled as a vector $\mathbf{V}=\left[V_{0}, \ldots V_{L-1}\right]$ of size $L$, whose coefficients are set to zero whenever their index exceeds the ICI spread. For instance, if we use LTE parameters with symbol duration $T=66.6 \mu$ s and cyclic prefix duration is $\Delta=4.69 \mu \mathrm{s}$, the ICI weight for OFDM when the timing offset if uniformly distributed in $[0 ; T+\Delta]$, is equal to:

$$
\begin{aligned}
\mathbf{V}_{\text {OFDM }} & =\left[6.89 \times 10^{-1}, 9.47 \times 10^{-2}, 2.37 \times 10^{-2}\right. \\
& 1.05 \times 10^{-2}, 5.9 \times 10^{-3}, 3.8 \times 10^{-3} \\
& \left.2.6 \times 10^{-3}, 1.9 \times 10^{-3}, 1.5 \times 10^{-3}, 1.12 \times 10^{-3}\right]
\end{aligned}
$$

Similarly, the ICI weights if we use FBMC as the multicarrier modulation with PHYDYAS filter [6], and if the timing offset is uniformly distributed in $[0 ; T]$, is given by:

$$
\mathbf{V}_{\mathrm{FBMC}}=\left[8.23 \times 10^{-1}, 8.81 \times 10^{-2}\right]
$$

These expressions imply that with FBMC, ICI is generated in only one adjacent subcarrier, whereas with OFDM, 9 adjacent subcarriers are subject to ICI when a transmitter is active in a single subcarrier.

Vector $\mathbf{V}$ is inserted in the $L \times L$ matrix $\overline{\mathbf{G}}_{k q}$ representing the channel gains from transmitter $q$ to receiver $k$ as follows:

$$
\left\{\begin{array}{l}
\overline{\mathbf{G}}_{k q}(i, j)=g_{k q}(j) V_{|i-j|} \quad \forall k \neq q \\
\overline{\mathbf{G}}_{k k}(i, j)=g_{k k}(j) \delta_{i-j} \quad \forall k
\end{array}\right.
$$

where $g_{k q}(j)$ is the channel gain from transmitter $q$ to receiver $k$ in subcarrier $j$ and $\delta_{x}$ is the Kronecker delta. The global channel gain matrix of size $K L \times K L$ is then:

$$
\tilde{\mathbf{G}}=\left[\begin{array}{cccc}
\overline{\mathbf{G}}_{00} & \overline{\mathbf{G}}_{01} & \ldots & \overline{\mathbf{G}}_{0(K-1)} \\
\overline{\mathbf{G}}_{10} & \overline{\mathbf{G}}_{11} & \ldots & \overline{\mathbf{G}}_{1(K-1)} \\
\vdots & & \ddots & \vdots \\
\overline{\mathbf{G}}_{(K-1) 0} & \overline{\mathbf{G}}_{(K-1) 1} & \ldots & \overline{\mathbf{G}}_{(K-1)(K-1)}
\end{array}\right]
$$

Let $\tilde{\mathbf{p}}$ be the stacked $K L \times 1$ power vector, where $\tilde{P}_{k}^{l}=$ $\tilde{\mathbf{p}}(l+k L)$ is the power transmitted by D2D transmitter $k$ in subcarrier $l$. Then the SINR at D2D receiver $k$ in subcarrier $l$ is:

$$
\operatorname{SINR}_{k}^{l}=\frac{\tilde{G}_{l k}^{l k} \tilde{P}_{k}^{l}}{n_{k}^{l}+\sum_{\substack{j=0 \\ j \neq k}}^{K-1} \sum_{l^{\prime}=0}^{L-1} \tilde{G}_{l^{\prime} j}^{l k} \tilde{P}_{j}^{l^{\prime}}}
$$

where $n_{k}^{l}$ is the noise at receiver $k$ in subcarrier $l$.

The per-RB allocation constraint implies that the same power should be allocated in all the subcarriers of a given $\mathrm{RB}$, for each user. Let $\mathcal{R}_{r}$ be the index set of subcarriers corresponding to RB $r$ and $P_{k}^{r}$ be the per-RB power allocated by transmitter $k$ in $\mathrm{RB} r$. Then the following constraint holds:

$$
\tilde{P}_{k}^{l}=P_{k}^{r}, \forall r \in \mathcal{R}_{r}
$$

\section{D2D MULTIPLEXING MAXIMIZATION}

\section{A. Feasibility criterion}

We consider the multiplexing problem of all $K$ devices on $N \mathrm{RB}$, subject to a minimum SINR constraint. Let $a_{k}^{r}$ be a binary value $a_{k}^{r} \in\{0,1\}$. Let $\gamma$ be the target SINR per subcarrier. If $a_{k}^{r}=1$, then D2D pair $k$ is active in RB $r$ with its SINR in all subcarriers $l \in \mathcal{R}_{r}$ larger than $\gamma$. Otherwise, $a_{k}^{r}=0$ and D2D pair is not allocated in RB $r$. The objective of RB allocation is to determine the vector a such that $a(r+k N)=a_{k}^{r}$ providing the optimum value of a given optimization problem. Vector a must be optimized considering co-channel and inter-channel interference coming from all interfering D2D pairs.

The SINR condition is written as follows, for $r \in$ $\{0, \ldots, N-1\}, l \in \mathcal{R}_{r}, k \in\{0, \ldots, K-1\}$ :

$$
\operatorname{SINR}_{k}^{l} \geq a_{k}^{r} \gamma
$$

The set of constraints (7) is equivalent to $K L$ equations:

$$
\tilde{P}_{k}^{l}-a_{k}^{r} \gamma \sum_{\substack{j=0 \\ j \neq k}}^{K-1} \sum_{l^{\prime}=0}^{L-1} \frac{\tilde{G}_{l^{\prime} j}^{l k}}{\tilde{G}_{l k}^{l k}} \tilde{P}_{j}^{l^{\prime}} \geq \frac{a_{k}^{r} \gamma n_{k}^{l}}{\tilde{G}_{l k}^{l k}}
$$

In order to obtain a feasible solution to equations (8), the allocation variable must be set per subcarrier ã such that $\tilde{a}(l+k L)=a_{k}^{l}$. The per-RB allocation constraint is written as: $\tilde{a}_{k}^{l}=a_{k}^{r}, \forall l \in \mathcal{R}_{r}$. Our objective is to maximize $\sum_{k=0}^{K-1} \sum_{l=0}^{L-1} \tilde{a}_{k}^{l}$ subject to the set of SINR constraints. We can notice that at optimal value of the allocation vector $\tilde{\mathbf{a}}$, all inequalities become equalities in the SINR constraints. Then the $K L$ equations can be written with matrix notations as follows:

$$
\left(\mathbf{I}_{K L}-\boldsymbol{\Gamma} \tilde{\mathbf{G}}_{\mathrm{dir}}^{-1} \tilde{\mathbf{G}}_{\text {int }}\right) \tilde{\mathbf{p}}=\tilde{\mathbf{G}}_{\mathrm{dir}}^{-1} \boldsymbol{\Gamma} \boldsymbol{n}
$$

where:

- $\tilde{\mathbf{G}}_{\mathrm{dir}}$ is the $K L \times K L$ diagonal matrix extracted from $\tilde{\mathbf{G}}$ by only taking its diagonal values. It thus corresponds to all direct channel gains.

- $\tilde{\mathbf{G}}_{\text {int }}=\tilde{\mathbf{G}}-\tilde{\mathbf{G}}_{\text {dir }}$ is the $K L \times K L$ matrix of interfering channel gains.

- $\boldsymbol{\Gamma}$ is a diagonal $K L \times K L$ matrix such that $\boldsymbol{\Gamma}(l+k L, l+$ $k L)=\tilde{a}_{k}^{l} \gamma$

- $\boldsymbol{n}$ is the $K L \times 1$ noise vector with $\boldsymbol{n}(l+k L)=n_{k}^{l}$,

To simplify notations, in the following, we note $\boldsymbol{\Psi}=$ $\boldsymbol{\Gamma} \tilde{\mathbf{G}}_{\text {dir }}^{-1} \tilde{\mathbf{G}}_{\text {int }}$. By Perron-Frobenius therorem, a feasible positive power allocation leading to $\tilde{a}_{k}^{l} \gamma$ for all users and subcarriers $(k, l)$ exists if and only if the spectral radius of matrix $\boldsymbol{\Psi}$, is strictly less than 1 [7]. The spectral radius is noted $\rho(\boldsymbol{\Psi})$ and is defined as the largest absolute value of the eigenvalues of matrix $\Psi$.

The maximum multiplexing problem aims at maximizing the number of D2D pairs multiplexed on the RB. It is written as follows:

$$
\begin{array}{ll}
\arg & \max _{\tilde{\mathbf{a}} \in\{0,1\}^{K \times L}} \sum_{k=0}^{K-1} \sum_{l=0}^{L-1} \tilde{a}_{k}^{l} \\
\text { s. t. } & \rho(\boldsymbol{\Psi})<1 \\
\text { s. t. } & \tilde{a}_{k}^{l}=a_{k}^{r}, \forall l \in \mathcal{R}_{r}, \forall k \in\{0, \ldots, K-1\}
\end{array}
$$


This problem is highly complex since there are $2^{K N}$ combinations in vector a and for each combination, computing the spectral radius of $\boldsymbol{\Psi}$ requires $\mathcal{O}\left((K L)^{3}\right)$ operations. The combinations can of course be ordered so as to test those with largest number of active D2D pairs at first, and stop as soon as one valid combination is obtained. However, in some cases, the number of active D2D pairs that allow to fulfill the spectral radius criterion may be low due to high interference, and the number of tested combinations may increase in such a way that complexity eventually becomes prohibitive.

In order to override these limitations, we propose two heuristics of lower complexity based on the Frobenius and the infinity norm. They are based on the following property of the spectral radius: the spectral radius is upper bounded by any norm of matrix $\Psi$.

\section{B. Heuristic based on Frobenius norm}

We first consider the Frobenius norm $\|\cdot\|_{F}$ and impose the constraint: $\|\boldsymbol{\Psi}\|_{F}^{2}<1$. This constraint implies that $\|\boldsymbol{\Psi}\|_{F}<1$. Then as $\rho(\Psi)<\|\Psi\|_{F}$, the spectral radius constraint $\rho(\Psi)<$ 1 to ensure multiplexing feasibility is fulfilled. The following problem is consequently studied:

$$
\begin{aligned}
& \arg \max _{\tilde{\mathbf{a}} \in\{0,1\}^{K \times L}} \sum_{k=0}^{K-1} \sum_{l=0}^{L-1} \tilde{a}_{k}^{l} \\
& \text { s. t. }\|\boldsymbol{\Psi}\|_{F}^{2}<1 \\
& \text { s. t. } \tilde{a}_{k}^{l}=a_{k}^{r}, \forall l \in \mathcal{R}_{r}, \forall k \in\{0, \ldots, K-1\}
\end{aligned}
$$

The solution of problem (11) provides a lower bound in terms of multiplexing on the solution of the initial problem (10).

The squared Frobenius norm of matrix $\Psi$ is:

$$
\begin{aligned}
\|\boldsymbol{\Psi}\|_{F}^{2} & =\sum_{k=0}^{K-1} \sum_{l=0}^{L-1} \sum_{j=0}^{K-1} \sum_{l^{\prime}=0}^{L-1}(\boldsymbol{\Psi})^{2}\left(l+k L, l^{\prime}+j L\right) \\
& =\gamma^{2} \sum_{k=0}^{K-1} \sum_{l=0}^{L-1} \tilde{a}_{k}^{l} \sum_{\substack{j=0 \\
j \neq k}}^{K-1} \sum_{l^{\prime}=0}^{L-1}\left(\frac{\tilde{\mathbf{G}}\left(l+k L, l^{\prime}+j L\right)}{\tilde{\mathbf{G}}(l+k L, l+k L)}\right)^{2}
\end{aligned}
$$

since $\left(\tilde{a}_{k}^{l}\right)^{2}=\tilde{a}_{k}^{l}$. Moreover, by including constraint $\tilde{a}_{k}^{l}=$ $a_{k}^{r} \forall l \in \mathcal{R}_{r}$ in equation (12), the squared Frobenius norm becomes:

$$
\|\Psi\|_{F}^{2}=\gamma^{2} \sum_{k=0}^{K-1} \sum_{r=0}^{N-1} a_{k}^{r} \sum_{l \in \mathcal{R}_{r}} \sum_{\substack{j=0 \\ j \neq k}}^{K-1} \sum_{l^{\prime}=0}^{L-1}\left(\frac{\tilde{\mathbf{G}}\left(l+k L, l^{\prime}+j L\right)}{\tilde{\mathbf{G}}(l+k L, l+k L)}\right)^{2}
$$

Let us define

$$
\beta_{k}^{r}=\sum_{l \in \mathcal{R}_{r}} \sum_{\substack{j=0 \\ j \neq k}}^{K-1} \sum_{l^{\prime}=0}^{L-1}\left(\frac{\tilde{\mathbf{G}}\left(l+k L, l^{\prime}+j L\right)}{\tilde{\mathbf{G}}(l+k L, l+k L)}\right)^{2}
$$

The optimization problem (11) is finally written as:

$$
\begin{aligned}
& \arg \max _{\mathbf{a} \in\{0,1\}^{K \times N}} \sum_{k=0}^{K-1} \sum_{r=0}^{N-1} a_{k}^{r} \\
& \text { s. t. } \gamma^{2} \sum_{k=0}^{K-1} \sum_{r=0}^{N-1} \beta_{k}^{r} a_{k}^{r}<1
\end{aligned}
$$

This is a binary knapsack problem with non-integer weights $\boldsymbol{\beta}$. The variables set has been reduced to $K N$ binary elements. This problem can be solved with linear integer programming techniques of pseudo-polynomial complexity [8].

\section{Heuristic based on infinity norm}

The second heuristic is obtained by using the infinity norm $\|\cdot\|_{\infty}$ as an upper bound to the spectral radius. The heuristic providing a lower bound to the solution of the initial problem is:

$$
\begin{array}{ll}
\arg \max _{\tilde{\mathbf{a}} \in\{0,1\}^{K \times L}} \sum_{k=0}^{K-1} \sum_{l=0}^{L-1} \tilde{a}_{k}^{l} \\
\text { s. t. }\|\boldsymbol{\Psi}\|_{\infty}<1 \\
\text { s. t. } \tilde{a}_{k}^{l}=a_{k}^{r}, \forall l \in \mathcal{R}_{r}, \forall k \in\{0, \ldots, K-1\}
\end{array}
$$

The infinity norm $\|\Psi\|_{\infty}$ is defined as follows:

$$
\begin{aligned}
\|\Psi\|_{\infty} & =\max _{\substack{0 \leq k \leq K-1 \\
0 \leq l \leq L-1}} \sum_{j=0}^{K-1} \sum_{l^{\prime}=0}^{L-1}(\boldsymbol{\Psi})\left(l+k L, l^{\prime}+j L\right) \\
& =\max _{\substack{0 \leq k \leq K-1 \\
0 \leq l \leq L-1}} \sum_{\substack{j=0 \\
j \neq k}}^{K-1} \sum_{l^{\prime}=0}^{L-1} \tilde{a}_{k}^{l} \gamma \frac{\tilde{\mathbf{G}}\left(l+k L, l^{\prime}+j L\right)}{\tilde{\mathbf{G}}(l+k L, l+k L)}
\end{aligned}
$$

To simplify notations, let us define $E_{k}^{l}$ :

$$
E_{k}^{l}=\gamma \sum_{\substack{j=0 \\ j \neq k}}^{K-1} \sum_{l^{\prime}=0}^{L-1} \frac{\tilde{\mathbf{G}}\left(l+k L, l^{\prime}+j L\right)}{\tilde{\mathbf{G}}(l+k L, l+k L)}
$$

Constraint $\|\boldsymbol{\Psi}\|_{\infty}<1$ is then equivalent to $\tilde{a}_{k}^{l} E_{k}^{l}<1$ for all $(k, l)$. This constraint is only fulfilled if $\tilde{a}_{k}^{l}=0$ whenever $E_{k}^{l} \geq 1$. This solution is optimal since all values of $\tilde{a}_{k}^{l}$ are equal to 1 , except those that would violate condition $\|\Psi\|_{\infty}<1$. The sum of $\tilde{a}_{k}^{l}$ under this constraint is then at its maximum. Finally, since resource allocation is performed per $\mathrm{RB}$, if $E_{k}^{l} \geq 1$ on at least one subcarrier $l \in \mathcal{R}_{r}$, then $\mathrm{D} 2 \mathrm{D}$ pair $k$ is not multiplexed on $\mathrm{RB} r$. The infinity norm provides a distributed algorithm: at each receiver, $E_{k}^{l}$ is computed depending on local information, that is deduced from the received interfering channel gains. This feature is quite appealing for future implementation of this algorithm in practical D2D networks.

After RB allocation has been performed, the power vector is directly obtained by inverting matrix $\left(\mathbf{I}_{K L}-\mathbf{\Psi}\right)$ in equation (9) :

$$
\tilde{\mathbf{p}}^{*}=\left(\mathbf{I}_{K L}-\boldsymbol{\Psi}\right)^{-1} \tilde{\mathbf{G}}_{\mathrm{dir}}^{-1} \boldsymbol{\Gamma} \boldsymbol{n}
$$

Since this power vector is defined per subcarrier, the transmit power of transmitter $k$ in $\mathrm{RB} r$ is finally chosen as the maximum transmit power of $k$ in all subcarriers of $\mathrm{RB} r$.

\section{D2D DISTRIBUTED SUM SINR MAXIMIZATION}

We now assume that a can take any integer value. Then, the SINR per D2D receiver and RB is a multiple of $\gamma$. With the same reasoning as in the previous section, all D2D pairs $k$ can jointly achieve their SINR $a_{k}^{r} \gamma$ in all subcarriers of RB $r$ if $\rho(\boldsymbol{\Psi})$ is lower than 1 . Then, the maximum sum SINR 
optimization problem with SINR multiple of $\gamma$ is written as follows:

$$
\begin{array}{ll}
\arg & \max _{\tilde{\mathbf{a}} \in \mathbb{N}^{K \times L}} \sum_{k=0}^{K-1} \sum_{l=0}^{L-1} \tilde{a}_{k}^{l} \\
\text { s. t. } & \rho(\boldsymbol{\Psi})<1 \\
\text { s. t. } & \tilde{a}_{k}^{l}=a_{k}^{r}, \forall l \in \mathcal{R}_{r}
\end{array}
$$

In order to obtain a distributed criterion, the spectral radius is upper-bounded by the infinity norm. The optimization problem becomes:

$$
\begin{aligned}
& \arg \max _{\tilde{\mathbf{a}} \in \mathbb{N} K \times L} \sum_{k=0}^{K-1} \sum_{l=0}^{L-1} \tilde{a}_{k}^{l} \\
& \text { s. t. } \max _{\substack{0 \leq k \leq K-1 \\
0 \leq l \leq L-1}} \tilde{a}_{k}^{l} E_{k}^{l}<1 \\
& \text { s. t. } \tilde{a}_{k}^{l}=a_{k}^{r}, \forall l \in \mathcal{R}_{r}
\end{aligned}
$$

where $E_{k}^{l}$ is defined by eq. (18). The first constraint is equivalent to the following set of constraints:

$$
\tilde{a}_{k}^{l} E_{k}^{l}<1 \forall(k, l) \in\{0, \ldots, K-1\} \times\{0, \ldots, L-1\}
$$

Let $\epsilon$ be a very small positive value. The optimal integer value for $\tilde{a}_{k}^{l}$ is equal to:

$$
\tilde{a}_{k}^{l}=\left\lfloor\frac{1-\epsilon}{E_{k}^{l}}\right\rfloor
$$

Then $a_{k}^{r}$ is obtained as the minimum of all $\tilde{a}_{k}^{l}, \forall l \in \mathcal{R}_{r}$, so that the same minimum SINR is reached on all subcarriers $l$ in $\mathrm{RB} r$.

$$
a_{k}^{r}=\min _{l \in \mathcal{R}_{r}}\left\{\tilde{a}_{k}^{l}\right\}
$$

The transmit power values are then computed with eq. (19).

\section{Simulation Results}

We consider a varying number of D2D pairs $K=12$ to 24 whose transmitters are uniformly located in a cellular area of radius $500 \mathrm{~m}$. Each D2D receiver is uniformly located at most at $50 \mathrm{~m}$ from its D2D transmitter. Thermal noise $n_{0}$ is white additive Gaussian with power spectral density -174 $\mathrm{dBm} / \mathrm{Hz}$. Shadowing follows a log-normal distribution with standard deviation equal to $4 \mathrm{~dB}$. $\gamma$ is equal to $10 \mathrm{~dB}$. The path loss model is small cell's path loss: $L_{\mathrm{dB}}=140+36.8 \log _{10}(d)$, where $d$ is expressed in $\mathrm{km}$. Multi-path fading is computed with Indoor Channel-B model [9]. The number of RB is $N=$ 25 , which in LTE corresponds to a $5 \mathrm{MHz}$ bandwidth. The ICI weights were given in Section II.

\section{A. Maximum multiplexing problem}

The performances of maximum multiplexing heuristics are compared with a Frequency Division Multiple Access (FDMA) solution, where each D2D pairs gets the same amount of $\mathrm{RB}$, and $\mathrm{RB}$ are randomly allocated to D2D pairs. Each D2D transmitter then uses equal power allocation on its allocated RB.

Fig. 1 shows that the average data rate is greatly improved by multiplexing. Moreover, using the infinity norm instead of the Frobenius norm is also beneficial, in terms of multiplexing (see Fig. 2) as well as in average data rate. When the Frobenius norm is used, the number of multiplexed pairs depends on

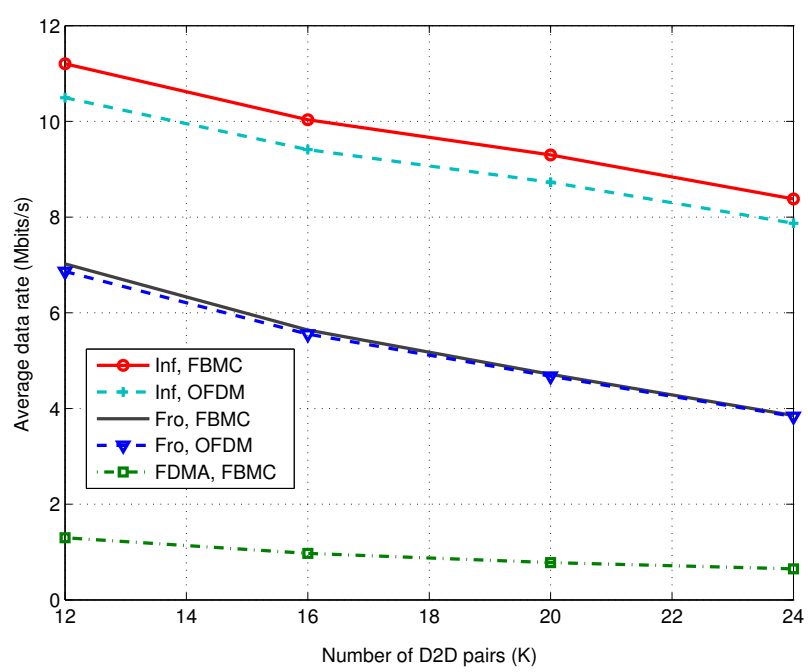

Fig. 1. Average rate per D2D pair, multiplexing maximization

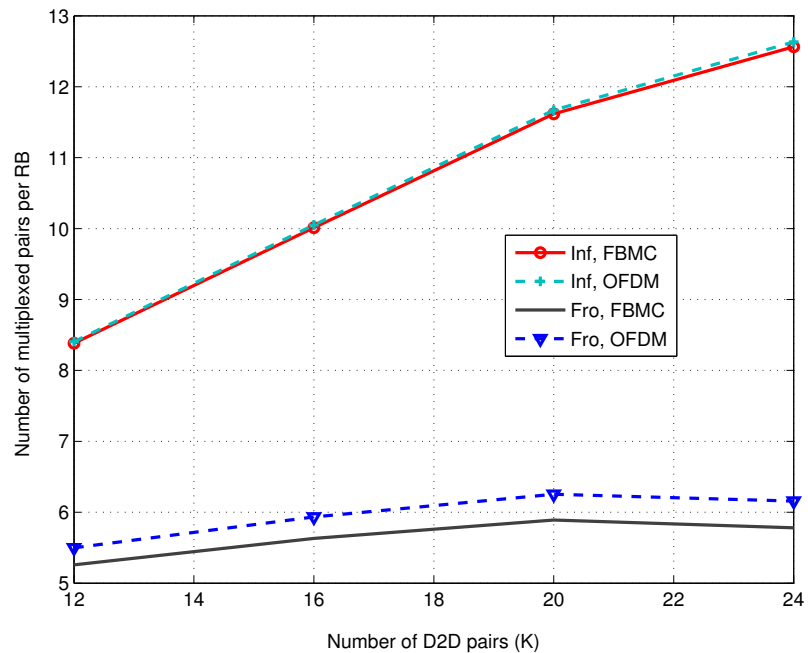

Fig. 2. Average number of D2D pairs multiplexed per RB, multiplexing maximization

$\|\Psi\|_{F}{ }^{2}$, which is lower than $\|\Psi\|_{F}$ when this latter is lower than 1. Consequently, constraint $\|\Psi\|_{F}{ }^{2}<1$ is too severe and highly decreases the multiplexing gain. It can be further inferred that any $l$-norm with $l>2$ would lead to even worse results. The infinity norm and the 1-norm are the only norms that allow avoiding this issue. Yet, the infinity norm has the great advantage of leading to a distributed algorithm, contrary to the 1-norm. The average data rate achieved with the infinity norm is higher with FBMC than with OFDM because there is no overhead due to cyclic prefix. Since power values and interferences are very low, the influence of ICI is almost negligible in this scenario, contrary to what was obtained with larger rates and power control in [4] $]^{1}$.

\section{B. Maximum sum rate problem}

Fig. 3 and 4 show the Cumulative Distribution Function (CDF) of the average data rate and of the transmit power, respectively, when the maximum sum rate problem is studied. The achieved data rate is highly unfair because some D2D

\footnotetext{
${ }^{1}$ Moreover, the same algorithm has been tested with Fast Fourier TransformFBMC (FFT-FBMC) [10], that has even better frequency localization than FBMC, and similar results have been obtained.
} 


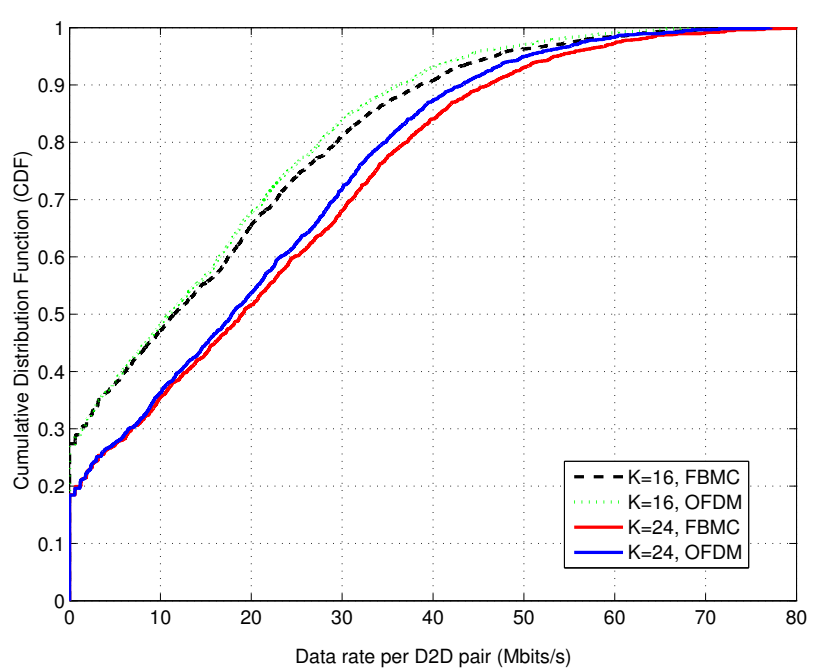

Fig. 3. Cumulative Distribution Function of the data rate, sum SINR maximization

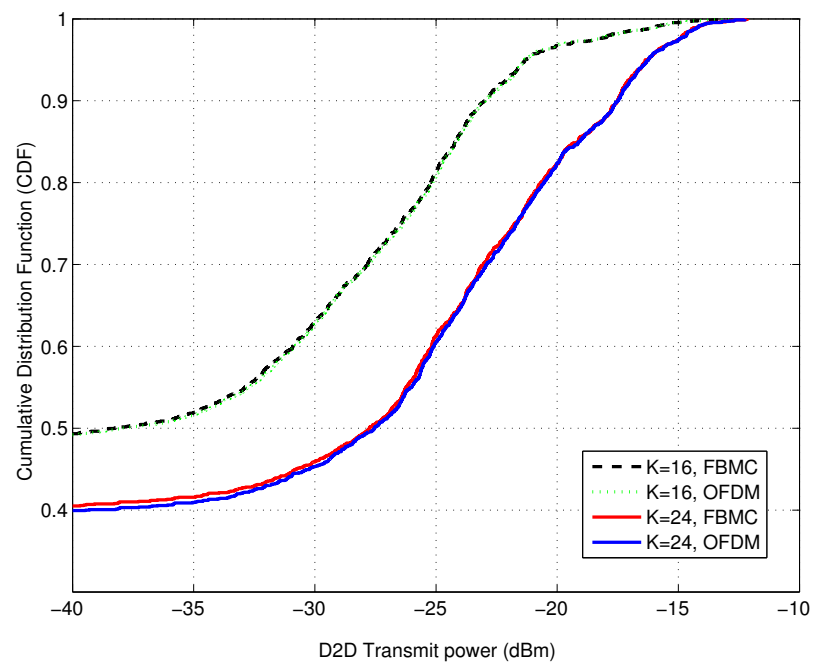

Fig. 4. Cumulative Distribution Function of the transmit power, sum SINR maximization

pairs obtain very high values of $a_{k}^{r}$, while some others obtain no RB at all. High data rates are achieved for the D2D pairs that are almost isolated. The average transmit power increases with the number of D2D pairs due to larger interference values that must be compensated for by power. However, the transmit power remains very low thanks to small transmitter-receiver distance.

\section{Distance to the optimal solution}

Finally, in this section, we compare the average data rate obtained with the infinity norm criterion, by solving problem (21), and the optimal maximum multiplexing solution achieved when solving the initial problem (10) using the spectral radius. We do not test the Frobenius norm criterion since it was shown to be less efficient than the infinity norm criterion. Due to the large complexity of solving (10) when the number of combinations to be tested is large (which corresponds to high interference cases and takes place when some D2D pairs are close), lower parameters are set: the number of $\mathrm{RB}$ is limited to 6 and the number of D2D pairs varies from 4 to 6 . Only the FBMC case is evaluated, but similar results would be obtained with OFDM. Table I shows that the distance in terms of average data rate between the optimal solution and the solution obtained with the infinity norm heuristic is quite low. It is limited to $9 \%$. With larger number of D2D pairs and of RB, this gap would most probably increase, but then the complexity of solving the initial problem becomes highly impractical.

TABLE I

AVERAGE DATA RATE DEPENDING ON THE CHOSEN ALGORITHM (MBITS/S)

\begin{tabular}{|l|c|c|c|}
\hline$K$ & Optimal algorithm & Infinity norm criterion & Rate decrease \\
\hline 4 & 3.62 & 3.30 & $8.98 \%$ \\
\hline 5 & 3.66 & 3.35 & $8.35 \%$ \\
\hline 6 & 3.51 & 3.22 & $8.31 \%$ \\
\hline
\end{tabular}

\section{CONCLUSIONS}

This paper has investigated the maximum multiplexing and the maximum sum SINR problems for asynchronous D2D communications. The system model including ICI has been obtained, and three heuristics have been proposed for RB allocation. The heuristic based on the infinity norm provides far larger multiplexing gains and data rates than that based on the Frobenius norm. The achieved data rates are within a reasonable distance to the ones obtained by solving the optimal initial problem, that should not be used due to its large complexity. Moreover, very high average data rates can be obtained when considering the maximum sum SINR problem, which emphasizes the benefits of D2D communications.

\section{ACKNOWLEDGEMENT}

This work was partially funded through French National Research Agency (ANR) project ACCENT5 with grant agreement code: ANR-14-CE28-0026-02.

\section{REFERENCES}

[1] X. Cai, J. Zheng, and Y. Zhang, "A graph-coloring based resource allocation algorithm for D2D communication in cellular networks ", in Proc. of ICC 2015, London, UK, May 2015.

[2] R. Zhang, X. Cheng, L. Yang, and B. Jiao, "Interference graph-based resource allocation (InGRA) for D2D communications underlaying cellular networks ," IEEE Trans. Vehicular Technology, vol. 64, no. 8, pp. 3844-3850, Aug. 2015.

[3] T. D. Hoang, L.B. Le, and T. Le-Ngoc, "Resource allocation for D2D communication underlaid cellular networks using graph-based approach," IEEE Trans. Wireless Communications, vol. 15, no. 10, pp. 7099-7113, Oct. 2016.

[4] M. Pischella, R. Zakaria, and D. Le Ruyet, "Resource Block level power allocation in asynchronous multi-carrier D2D communications ," IEEE Communication Letters, vol. 21, no. 4, pp. 813-816, Apr. 2017.

[5] Y. Medjahdi, M. Terre, D. Le Ruyet, D. Roviras, J. A. Nossek, and L. Baltar, "Inter-cell Interference Analysis for OFDM / FBMC Systems," in Proc. of 10th IEEE Signal Processing Workshop (SPAWC 2009), Perugia, Italy, June 2009.

[6] M. G. Bellanger, "Specification and Design of a Prototype Filter for Filter Bank based Multicarrier Transmission," in Proc. of ICASSP 2001, Salt Lake City, UT, USA, May 2001.

[7] M. Pischella and J-C. Belfiore, "Distributed resource allocation for rateconstrained users in multi-cell OFDMA networks," IEEE Communication Letters, vol. 12, no. 4, pp. 250-252, Apr. 2008.

[8] G.L. Nemhauser L. A Wolsey, Integer and Combinatorial Optimization., Wiley-Interscience, New York, 199.

[9] "Guidelines for evaluation of radio transmission technologies for IMT2000," ITU-R Recommendation M.1225, 1997.

[10] R. Zakaria and D. Le Ruyet, "A novel fbmc scheme for spatial multiplexing with maximum likelihood detection," in 2010 7th International Symposium on Wireless Communication Systems, Sept 2010, pp. 461465 . 